\title{
Transverse colon schwannoma treated by endoscopic mucosal resection: A case report
}

\author{
DARYL RAMAI ${ }^{1,2}$, JONATHAN LAI $^{1}$, KINESH CHANGELA $^{2}$, \\ MADHAVI REDDY ${ }^{1}$ and GHULAMULLAH SHAHZAD ${ }^{2}$ \\ ${ }^{1}$ Department of Anatomical Sciences, St. George's University, Grenada, West Indies; ${ }^{2}$ Division of Gastroenterology, \\ The Brooklyn Hospital Center-Clinical Affiliate of Mount Sinai Hospital, New York, NY, USA
}

Received April 11, 2017; Accepted August 25, 2017

DOI: $10.3892 / \mathrm{mco} .2017 .1418$

\begin{abstract}
Gastrointestinal (GI) schwannoma is one of the rarest tumors of the GI tract. We herein describe the case of a 70-year-old female patient who presented for surveillance colonoscopy. The examination detected a 1-cm polyp that was removed by snare cautery polypectomy. Immune and histochemical staining revealed spindle cells that were positive for S-100 and vimentin, but negative for CD34 and smooth muscle actin, consistent with GI schwannoma. This case is noteworthy as GI schwannomas usually present in the stomach, making the finding of a colonic schwannoma of clinical interest. Furthermore, the present case was treated by removing the tumor endoscopically, in contrast to more invasive methods.
\end{abstract}

\section{Introduction}

Gastric schwannoma is slow-growing mesenchymal neoplasm that originates from Schwann cells (1). This is a rare subgroup of gastrointestinal stromal tumors (GISTs), which have low malignant potential, and are clinically distinct from other non-epithelial tumors of the gastrointestinal (GI) tract, including leiomyoma, leiomyosarcoma and GI autonomic neurogenic tumors. GI schwannomas account for $\sim 2-6 \%$ of all submucosal tumors, and $\sim 60-70 \%$ occur within the stomach (2). While this type of tumor rarely occurs in the bowel, schwannomas may develop in any anatomical region. While the majority of schwannomas are benign, it is important to accurately identify these tumors as they may mimic other malignant lesions of the GI tract. This report presents a case of a schwannoma found at the transverse colon upon surveillance colonoscopy, which was treated by endoscopic mucosal resection.

Correspondence to: Dr Daryl Ramai, Department of Anatomical Sciences, St. George's University, True Blue, Grenada, West Indies E-mail: dramai@sgu.edu

Key words: schwannoma, endoscopic mucosal resection, colon

\section{Case report}

A 70-year-old female patient was referred by her primary care physician for surveillance colonoscopy in January 2017. The patient reported no major symptoms, but complained of occasional episodes of diarrhea over the past month. The patient was a non-smoker and non-drinker, and review of the systems was negative for any weight loss. The finding on physical examination were unremarkable. The vital signs were stable, and the laboratory results were within normal limits. The patient underwent a colonoscopy, which revealed evidence of moderately severe diverticulosis in the sigmoid and descending colon. A single polyp, measuring $1 \mathrm{~cm}$ in size, was identified in the transverse colon. Saline was injected at the base to raise the polyp prior to removal (Fig. 1A). The polyp was completely removed by snare cautery polypectomy. To control bleeding, 2 clips were applied (Fig. 1B). Histological examination of the polyp showed a solid mass with peripheral colonic mucosa (Fig. 2A). Higher resolution revealed fascicles of spindle cells exhibiting nuclear palisading (Fig. 2B and C). Immunohistochemistry was positive for S-100 and vimentin, but negative for CD34 and smooth muscle actin, consistent with GI schwannoma. The patient made a good postoperative recovery. Consent was obtained from the patient regarding the publication of the case details and associated images.

\section{Discussion}

GI schwannoma is an extremely rare intestinal mesenchymal tumor that was first described by Daimaru et al in 1988. Unlike typical schwannomas, which arise from peripheral nerves of the skin, connective tissue and internal organs, GI schwannomas are considered to arise from the autonomous nervous system, more commonly from the Auerbach's plexus, and less frequently from the Meissner's plexus (1). Drawing from the few case reports and studies published on this subject, these cases may occur at any age, but are most frequently encountered in the fifth and sixth decades of life, with identical rates for men and women (2). GI schwannoma occurs most frequently in the stomach $(83 \%)$ and small intestine (13\%), making the finding of transverse colon schwannoma a very rare clinical entity (3). 

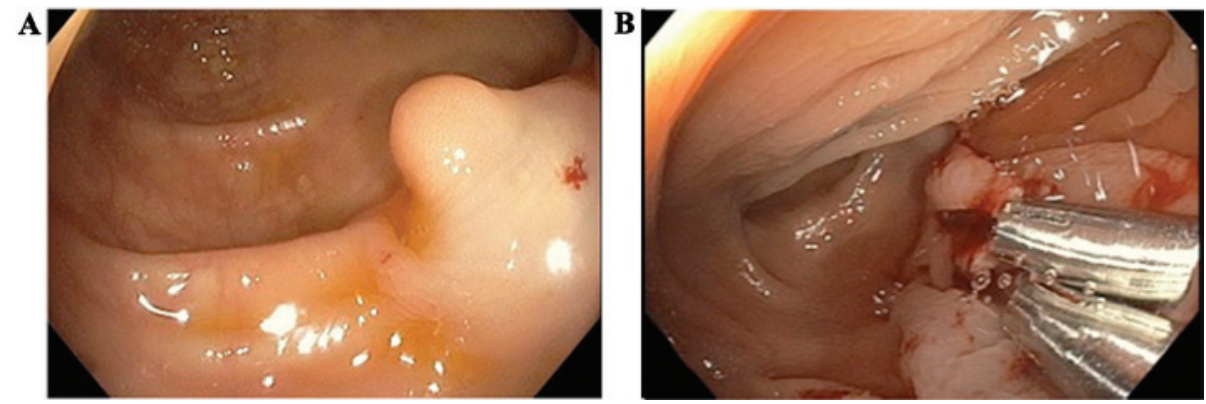

Figure 1. (A) The saline-injected polyp $(1 \mathrm{~cm})$ prior to removal. (B) Polyp completely removed by snare cautery polypectomy and two clips applied for bleeding control.

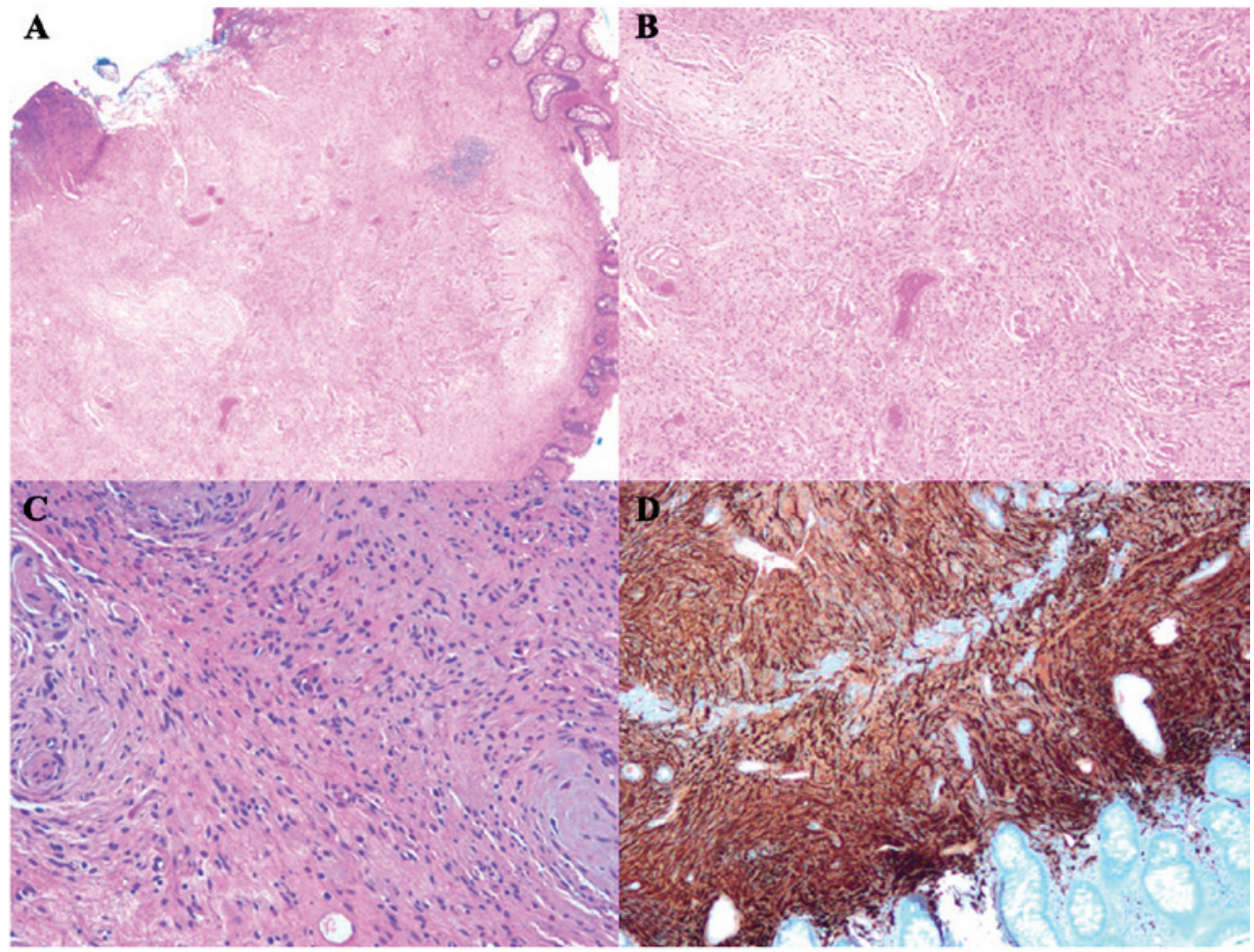

Figure 2. Hematoxylin and eosin staining. (A) Low-magnification view (x20) shows a solid mass with peripheral colonic mucosa; (B and C) the tumor was composed of fascicles of spindle cells exhibiting nuclear palisading (magnification, $\mathrm{x} 40$ and x100, respectively). (D) Immunohistochemistry was positive for S-100 (magnification, x100).

Neoplasms originating from the Auerbach's plexus typically protrude into the intestinal lumen and are characterized by a non-pedunculated oval-shaped mass; those arising from the Meissner's plexus are often similar to pedunculated polyps. Schwannomas are known to be benign neoplasms of ectodermal origin, which are characterized by a slow growth pattern with the capacity for malignant degeneration if not removed (4). This type of tumor typically manifests as a polyp that may ulcerate the mucosa, leading to non-specific symptoms, including abdominal pain with rectal bleeding, defecation disorders and colonic obstruction (5).

GI schwannoma is a unique clinical finding, which is not associated with systemic neurofibromatosis or von Recklinghausen's disease. Furthermore, while schwannomas are considered to be a subtype of GIST, they have unique histopathological and immunohistochemical characteristics, which are vital for accurate identification. Unlike GISTs, schwannomas are typically negative for CD117 (KIT), CD34 and actin, but positive for S-100 and vimentin (6). Schwannomas are histologically characterized by spiral-like forms consisting of densely arrayed spindle-shaped tumor cells, palisading arrangement, and loose reticular networks of tumor cells (7).

As regards the treatment method, a tumor size of $\geq 5 \mathrm{~cm}$ is considered to be a criterion for surgery, as recurrence and prognosis vary greatly if the tumor is $>5 \mathrm{~cm}$ in diameter (8). However, the benign nature of the tumor is responsible for the good prognosis of patients with schwannoma; recurrence and metastasis are considered rare events. Our patient was treated with endoscopic mucosal resection, a safe and minimally invasive technique used for removing lesions in the GI tract. The most frequent complication associated with this technique is bleeding; however, the rate of this complication is very low (9). 


\section{References}

1. Daimaru Y, Kido H, Hashimoto $\mathrm{H}$ and Enjoji M: Benign schwannoma of the gastrointestinal tract: A clinicopathologic and immunohistochemical study. Hum Pathol 19: 257-264, 1988.

2. Miettinen M, Sarlomo-Rikala M and Lasota J: Gastrointestinal stromal tumours. Ann Chir Gynaecol 87: 278-281, 1998.

3. Nonose R, Lahan AY, Valenciano Santos J and Martinez CA Schwannoma of the colon. Case Rep Gastroenterol 3: 293-299, 2009.

4. Lauwers GY, Erlandson RA, Casper ES, Brennan MF and Woodruff JM: Gastrointestinal autonomic nerve tumors: A clinicopathological, immunohistochemical, and ultrastructural study of 12 cases. Am J Surg Pathol 17: 887-897, 1993.

5. Kwon MS, Lee SS and Ahn GH: Schwannomas of the gastrointestinal tract: Clinicopathological features of 12 cases including a case of esophageal tumor compared with those of gastrointestinal stromal tumors and leiomyomas of the gastrointestinal tract. Pathol Res Pract 198: 605-613, 2002.
6. Zippi M, Pica R, Scialpi R, Cassieri C, Avallone EV and Occhigrossi G: Schwannoma of the rectum: A case report and literature review. World J Clin Cases 1: 49-51, 2013.

7. Hasegawa T, Tashiro T, Sekine S, et al: Pathology and classification of gastrointestinal submucosal tumors including GIST. Stomach Intestine 39: 396-404, 2004.

8. Jacobson BC, Hirsch MS, Lee JH, Van Dam J, Shoji B and Farraye FA: Multiple asymptomatic plexiform schwannomas of the sigmoid colon: A case report and review. Gastrointest Endosc 53: 801-804, 2001.

9. Bergmann U and Beger HG: Endoscopic mucosal resection for advanced non-polypoid colorectal adenoma and early stage carcinoma. Surg Endosc 17: 475-479, 2003. 